\title{
Early alert from the microbiology laboratory improves the outcome of elderly patients with Enterococcus spp. bloodstream infection: Results from a multicentre prospective study
}

\author{
M. Falcone ${ }^{\mathrm{a}, *}$, G. Tiseo ${ }^{\mathrm{b}}$, F. Dentali ${ }^{\mathrm{c}}$, E. Foglia $^{\mathrm{d}}$, M. Campanini ${ }^{\mathrm{e}}$, F. Menichetti $^{\mathrm{a}}$, \\ A. Mazzone ${ }^{\mathrm{f}}$, on behalf of the FADOI (Federazione delle Associazioni dei Dirigenti \\ Ospedalieri Internisti) and GISA (Italian Group for Antimicrobial Stewardship) \\ a Division of Infectious Diseases, Department of Clinical and Experimental Medicine, University of Pisa, Pisa, Italy \\ b Department of Internal Medicine and Medical Specialties, 'Sapienza' University of Rome, Rome, Italy \\ ' Department of Clinical Medicine, University of Insubria, Varese, Italy \\ d Centre for Research on Health Economics, Social and Health Care Management (CREMS), University Carlo Cattaneo-LIUC, Castellanza, Italy \\ e Internal Medicine Ward, Ospedale Maggiore della Carità, Novara, Italy \\ ${ }^{\mathrm{f}}$ Internal Medicine Ward, Ospedale Civile, Legnano, Italy
}

\section{A R T I C L E I N F O}

\section{Article history:}

Received 11 December 2018

Received in revised form 4 February 2019

Accepted 19 February 2019

Available online 27 February 2019

\section{Keywords:}

Enterococcus spp.

Bloodstream infection

Elderly patients

Microbiology laboratory

\begin{abstract}
A B S T R A C T
Objectives: This study describes the clinical features and outcomes of patients with bloodstream infection (BSI) due to Enterococcus spp. and identified factors predictive of mortality.

Methods: This analysis is part of a prospective multicentre observational study of consecutive hospitalised patients with BSI conducted from March 2012 to December 2012 in 31 internal medicine wards in Italy. Patients with enterococcal BSI were selected from the entire cohort. Patient characteristics, therapeutic interventions and outcome were reviewed. Cox regression analysis was performed to identify factors associated with in-hospital mortality. Hazard ratios (HRs) and 95\% interval confidences (CIs) were calculated.

Results: Among 533 patients with BSI, 41 (7.7\%) had BSI by Enterococcus spp. (28 Enterococcus faecalis, 4 Enterococcus faecium and 3 each of Enterococcus avium, Enterococcus casseliflavus and Enterococcus gallinarum). Six BSIs (14.6\%) were polymicrobial. Median (IQR) patient age was 73 (66-85.5) years. Inhospital mortality was $24.4 \%$. Polymicrobial infection ( $\mathrm{HR}=9.100,95 \% \mathrm{CI} 1.295-63.949 ; P=0.026)$, age $(\mathrm{HR}=1.261,95 \% \mathrm{CI} 1.029-1.546 ; P=0.025)$ and SOFA score $(\mathrm{HR}=1.244,95 \% \mathrm{CI} 1.051-1.474 ; P=0.011)$ were risk factors for in-hospital mortality. Conversely, receiving an alert from the microbiology laboratory before obtaining final antimicrobial susceptibility results was associated with survival $(\mathrm{HR}=0.073,95 \%$ CI 0.007-0.805; $P=0.033$ ).

Conclusion: BSI due to Enterococcus spp. in elderly patients is associated with high mortality. Polymicrobial infection, age and SOFA score are factors associated with poor outcome. Conversely, early alert from the microbiology laboratory improves patient survival.

(C) 2019 International Society for Chemotherapy of Infection and Cancer. Published by Elsevier Ltd. All rights reserved.
\end{abstract}

\section{Introduction}

Enterococcus spp. are an important cause of nosocomial and community-acquired infections both in the USA [1,2] and Europe [3]. Traditionally, Enterococcus faecalis has been considered as the leading infecting species. Moreover, in the last decades several Enterococcus spp. with novel mechanisms of acquired resistance to

\footnotetext{
* Corresponding author.

E-mail address: marco.falcone@unipi.it (M. Falcone).
}

antimicrobials have became more and more prevalent [4], and vancomycin-resistant enterococci (VRE) causing hospital outbreaks worldwide represent an important threat to patient care [5-8]. A large number of molecular epidemiological studies have been performed to obtain insights into the dissemination of VRE clones in and between hospitals, in farm animals and in healthy humans. It has been reported that $>80 \%$ of Enterococcus faecium isolates currently recovered from US hospitals are resistant to vancomycin, and virtually all of them (>90\%) exhibit ampicillin resistance [9]. Bloodstream infections (BSIs) due to vancomycinresistant $E$. faecium not only incur significant costs for healthcare 
systems but also place vulnerable patients at greater higher risk of fatal infection [10].

In-hospital mortality associated with enterococcal BSI varies greatly between studies depending on the type of patients, ward of hospitalisation and antimicrobial susceptibility profile [11,12]. In one study of patients with enterococcal bacteraemia, the crude 30 -day mortality rate was $23 \%$ with an attributable mortality rate of $6 \%$ [7], whereas in patients with VRE bacteraemia in another study the crude and attributable mortality rates were $50.2 \%$ and $30.3 \%$, respectively [13]. A recent study of patients with intensive care unit (ICU)-acquired enterococcal BSI showed that these infections are associated with increased case fatality but with low attributable mortality [14]. Infections such as bacteraemia and endocarditis can cause life-threatening disease with high mortality rates, especially in elderly patients with multiple comorbidities [2,15-17]. In these cases, prompt and accurate identification and early appropriate antibiotic therapy are essential to reduce mortality [18].

The aim of the current study was to describe the clinical features and outcomes of elderly patients with BSI due to Enterococcus spp. and to identify factors independently associated with mortality.

\section{Materials and methods}

\subsection{Study design}

This evaluation was performed as part of a prospective multicentre observational study of consecutive patients with BSI hospitalised in 31 internal medicine wards from 14 different Italian regions from 1 March 2012 to 31 December 2012 (SNOOPII study) $[19,20]$. A minimum of 15 patients were requested from each participating centre. All included patients were monitored daily from the onset of BSI until the end of hospitalisation. Approval of the study protocol was obtained from the institutional review boards at each hospital, which waived the requirement for obtaining informed consent. The study was conducted under the auspices of the Federation of Association of Executives of Hospital Internists (FADOI).

All consecutive patients aged $\geq 18$ years admitted to the hospital with BSI or those developing a BSI during hospitalisation were included in the study. Among them, all patients with BSI caused by Enterococcus spp. have been analysed in the current study.

In each centre, trained physicians prospectively collected information on patient characteristics, daily clinical events and illness characteristics and reported it on a standard clinical record form. More specifically, demographic data such as age and date of hospital admission were recorded. For each patient, the investigators recorded past medical history, reason for hospital admission and co-morbidities as assessed by the Charlson comorbidity index. Information about co-morbid medical conditions such as diabetes, cardiovascular disease, chronic obstructive pulmonary disease, liver disease and cancer was obtained through review of the medical records. Clinical data such as presence of fever, hypotension, tachycardia, tachypnoea, altered mental status and signs of organ failure at the onset of BSI were recorded. The presence of a medical device (peripherally inserted central catheter, dialysis catheter, central venous catheter or implanted line) and urinary catheter was recorded. Relevant therapies, including cytotoxic chemotherapy, systemic corticosteroids, immunosuppressive agents or radiation therapy, in the 3 months prior to BSI were documented. According to the National Healthcare Safety Network (NHSN), the probable source of BSI was assessed according to the available clinical and microbiological information and was classified by the investigators using the following categories: genitourinary tract; respiratory tract; gastrointestinal; skin and soft tissue; surgical site; line-related; endocarditis; or unknown [21]. Length of stay was calculated as the number of days from the date of hospital admission to the date of discharge. In addition, clinical management of indwelling catheters, initiation of antimicrobial therapy and various clinical outcomes related to the BSI were also recorded. Information about the reporting of positive blood cultures by the clinical microbiology laboratory (including time of blood culture collection, time of microbiology positivity and time of communication of the preliminary results to physicians) was collected in the study data form. Two ways of reporting of the blood cultures results were considered [22]:

- Early alert from the clinical microbiology laboratory: defined as a phone call from the microbiologist to the physician in charge about the identified species growing in the blood culture. The physician in charge was informed by telephone about the result of species identification before completion of antimicrobial susceptibility testing; and

- Conventional reporting: clinicians received information about the isolated organism only when the blood culture report was completed and returned from the laboratory to the hospital ward.

The time from blood culture collection to report of blood culture results was calculated and expressed in hours and was represented by (i) the time from blood culture collection and the phone call from the microbiologist if the early alert was received or (ii) the time from blood culture collection to the standard receipt of blood cultures when conventional reporting was performed.

\subsection{Definitions}

A BSI was defined according to the standard definitions of the US Centers for Disease Control and Prevention (CDC) [23]. BSI episodes were classified as: (i) nosocomial, if a positive blood culture was obtained from patients who had been hospitalised for $\geq 48 \mathrm{~h}$; (ii) healthcare-associated, if a positive blood culture was obtained from a patient at the time of hospital admission or within $48 \mathrm{~h}$ of admission if the patient (a) had received intravenous (i.v.) therapy at home or had self-administered i.v. medical therapy in the 30 days before the BSI, (b) had received wound care or specialised nursing care through a healthcare agency, family or friends, (c) had attended a hospital or haemodialysis clinic or received i.v. chemotherapy in the 30 days before the BSI, (d) was hospitalised in an acute-care hospital for $\geq 2$ days in the 90 days before the BSI or (e) resided in a nursing home or long-term care facility; and (iii) community-acquired, if a positive blood culture obtained at the time of hospital admission or within $48 \mathrm{~h}$ of hospital admission for patients who did not fit the criteria for healthcare-associated infection [24]. Neutropenia was defined as an absolute neutrophil count of $<500$ cells $/ \mathrm{mm}^{3}$ within 30 days before the BSI [25]. Immunosuppressive therapy was defined as the use of steroids (prednisolone $>0.5 \mathrm{mg} / \mathrm{kg} /$ day or equivalent for $>1$ month), chemotherapy or anti-tumour necrosis factor, cyclophosphamide, azathioprine, methotrexate, mycophenolate mofetil or calcineurin inhibitor therapy within the past 3 months. Chronic kidney disease was defined according to Kidney Disease Outcomes Quality Initiative (K/DOQI) guidelines [26].

\subsection{Microbiological analysis}

Microbiological examination was performed on sputum, urine and blood samples according to standards of practice. All isolates from patients were identified in a central microbiology laboratory 
from each study site using automated methods such as BacT/ ALERT $^{\circledR}$ (bioMérieux; Marcy-l'Étoile, France) and VITEK ${ }^{\circledR} 2$ broth microdilution (bioMérieux) according to European Committee on Antimicrobial Susceptibility Testing (EUCAST) interpretative standards [27]. In some, but not all, centres bacterial identification was carried out by matrix-assisted laser desorption/ionisation time-of-flight mass spectrometry (MALDI-TOF/MS) (VITEK ${ }^{\circledR}$ MS; bioMérieux).

\subsection{Aim of the study and statistical analysis}

The primary goal of this study was to describe the clinical features and outcomes of BSI episodes caused by Enterococcus spp. in patients hospitalised in Italian internal medicine wards and to identify risk factors for in-hospital mortality among these patients.

The original SNOOPII study expected the inclusion of a minimum of 15 consecutive patients with BSI from each participating centre. Of these cases, patients with enterococcal BSI were selected for the current study.

Statistical analysis was performed using commercially available statistical software packages [IBM SPSS Statistics v.20.0 (IBM Corp., Armonk, NY) and R v.3.0.2 (R Development Core Team, Vienna, Austria)]. Continuous variables were compared by Student's $t$-test if normally distributed and by Mann-Whitney $U$-test if nonnormally distributed. Categorical variables were evaluated using $\chi^{2}$ test or two-tailed Fisher's exact test. Values for continuous and categorical variables are expressed as the mean \pm standard deviation or median [interquartile range (IQR)] and as the percentage of the group from which they were derived, respectively. Multivariate analysis to identify independent risk factors for in-hospital mortality was performed using a logistic regression model.

Variables with a $P$-value of $<0.10$ and those considered clinically relevant according to available literature data were included in a stepwise Cox proportional hazard model. Independent variables with $P<0.05$ after stepwise elimination were included in the final multivariate regression. The hazard ratio (HR) and 95\% confidence interval $(\mathrm{CI})$ were calculated to evaluate the strength of any association. Statistical significance was established at $\leq 0.05$. All reported $P$-values are two-tailed.

\section{Results}

Fig. 1 shows the flow chart of the study. During the study period, a total of 533 patients with BSI were included in the SNOOPII study. Among these, 257 episodes (48.2\%) were caused by Gram-positive bacteria. Enterococcus spp. were responsible for 41 BSIs $(7.7 \%)$ observed in internal medicine wards. Overall, 35 (85.4\%) were monomicrobial and 6 (14.6\%) were polymicrobial. Of the 41 enterococcal BSI patients, $28(68.3 \%)$ had monomicrobial BSI caused by E. faecalis and 4 (9.8\%) had monomicrobial BSI caused by E. faecium. Three monomicrobial BSIs were caused by Enterococcus avium, Enterococcus casseliflavus and Enterococcus gallinarum, respectively. Of the six polymicrobial BSIs, two were associated with culture of methicillin-susceptible Staphylococcus aureus, one with Staphylococcus epidermidis (considered a skin contaminant), one with Klebsiella pneumoniae and two with Candida albicans. All cases, with the exception of one case of $E$. faecium-Candida co-infection, received appropriate therapy within the first $48 \mathrm{~h}$ from blood culture collection. In all cases, only the first set of blood cultures was positive for more than one microorganism, whilst the remaining blood cultures were positive only for Enterococcus spp.

Five isolates (12.2\%) were resistant to ampicillin and four isolates (9.8\%) were resistant to vancomycin (two E. faecium, one $E$. casseliflavus and one E. gallinarum).

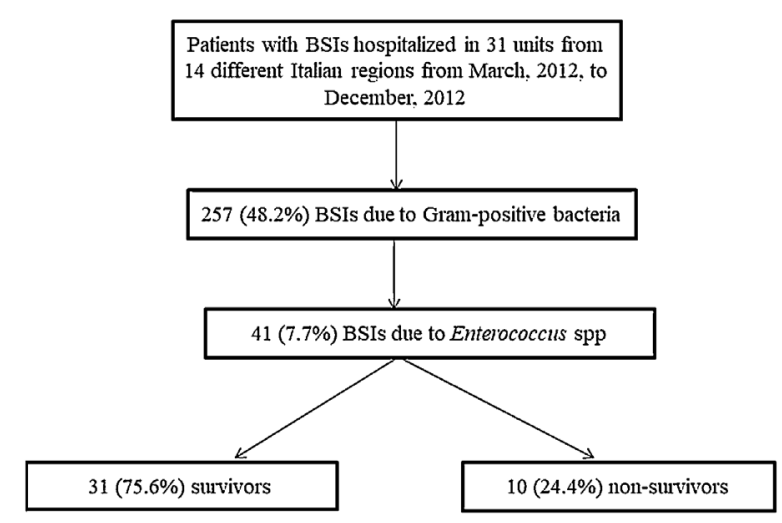

Fig. 1. Flow chart of study participation. BSI, bloodstream infection.

Table 1 shows the clinical features and outcomes of the 41 patients with enterococcal BSI. Patients included in the study were elderly with a median age of 73 years (IQR 66-85.5 years). The majority of episodes were community-onset, with 17 (41.5\%) healthcare-associated and 10 (24.4\%) community-acquired BSIs. The most common sources of infection were the genitourinary tract (34.1\%) and gastrointestinal tract (22.0\%), followed by primary BSI (19.5\%). Clinicians received an early alert from the microbiology laboratory for 20 patients (48.8\%). The median (IQR) time from blood culture collection to knowledge of the results was shorter in the case of early alert from microbiology compared with conventional reporting [ $39 \mathrm{~h}(24-48 \mathrm{~h})$ vs. $96 \mathrm{~h}(72-120 \mathrm{~h})$; $P<0.001]$. After receiving the phone call from microbiology, clinicians decided to change the antimicrobial therapy in 10 patients: 4 patients discontinued fluoroquinolones and started ampicillin plus aminoglycosides $(n=2)$, imipenem $(n=1)$ or piperacillin/tazobactam (TZP) $(n=1)$, respectively; 2 patients discontinued TZP and started linezolid; 3 patients discontinued cephalosporins and started teicoplanin $(n=2)$ or ampicillin $(n=1)$, respectively; and 1 patient added aminoglycosides to their ampicillin therapy. All patients who underwent a change in antimicrobial therapy after the early alert from laboratory survived (data not shown).

The in-hospital mortality rate was $24.4 \%$ (10/41). Table 2 shows a comparison between survivors and non-survivors. Non-survivors had a higher median age ( 84.5 years vs. 70 years; $P=0.022$ ), were more frequently affected by a healthcare-associated BSI (70.0\% vs. 32.3\%; $P=0.035$ ), had a higher median Sequential Organ Failure Assessment (SOFA) score at presentation (11 vs. $2 ; P<0.001)$ and were more commonly affected by multiple co-morbidities (median Charlson co-morbidity index, 4 vs. $3 ; P=0.049$ ). Conversely, among survivors, clinicians more frequently received an alert communication from the microbiology laboratory regarding the blood culture positivity ( $58.1 \%$ vs. $20.0 \% ; P=0.036$ ).

In the multivariate analysis (Table 3 ), polymicrobial infection $(\mathrm{HR}=9.100,95 \% \mathrm{CI} 1.295-63.949 ; P=0.026)$, age $(\mathrm{HR}=1.261,95 \% \mathrm{CI}$ $1.029-1.546 ; P=0.025)$ and SOFA score $(\mathrm{HR}=1.244,95 \%$ CI $1.051-$ $1.474 ; P=0.011)$ were risk factors for in-hospital mortality. Conversely, an alert from the clinical microbiology laboratory represented the only factor independently associated with survival $(\mathrm{HR}=0.073,95 \%$ CI $0.007-0.805 ; P=0.033)$.

\section{Discussion}

This study describes the epidemiology and clinical features of elderly patients with enterococcal BSI hospitalised in 31 different internal medicine wards. Moreover, factors independently associated with in-hospital mortality among these patients were 
Table 1

Clinical features and outcomes of patients with bloodstream infection (BSI) due to Enterococcus spp. $(N=41)$.

\begin{tabular}{|c|c|}
\hline & $n(\%)^{\mathrm{a}}$ \\
\hline \multicolumn{2}{|l|}{ Demographic characteristics } \\
\hline Age (years) [median (IQR)] & $73(66-85.5)$ \\
\hline Age $\geq 65$ years & $33(80.5)$ \\
\hline Male sex & $20(48.8)$ \\
\hline Hospitalisation in last 3 months & $20(48.8)$ \\
\hline Antibiotic treatment in last 3 months & $19(46.3)$ \\
\hline \multicolumn{2}{|l|}{ Means of acquisition } \\
\hline Community-acquired & $10(24.4)$ \\
\hline Healthcare-associated & $17(41.5)$ \\
\hline Nosocomial & $14(34.1)$ \\
\hline \multicolumn{2}{|l|}{ Co-morbid conditions } \\
\hline Cardiovascular disease & $28(68.3)$ \\
\hline Diabetes & $16(39.0)$ \\
\hline COPD & $11(26.8)$ \\
\hline Solid malignancy & $10(24.4)$ \\
\hline Chronic kidney disease & $9(22.0)$ \\
\hline Liver disease & $6(14.6)$ \\
\hline Neutropenia & $1(2.4)$ \\
\hline Charlson comorbidity index [median (IQR)] & $3(2-5)$ \\
\hline SOFA score [median (IQR)] & $3(1-8)$ \\
\hline \multicolumn{2}{|l|}{ Clinical manifestations } \\
\hline Fever $\left(>38^{\circ} \mathrm{C}\right)$ & $38(92.7)$ \\
\hline Hypotension ${ }^{\mathrm{b}}$ & $7(17.1)$ \\
\hline Tachycardia & $30(73.2)$ \\
\hline Tachypnoea & $17(41.5)$ \\
\hline $\mathrm{PaO}_{2} / \mathrm{FiO}_{2}<300$ & $9(22.0)$ \\
\hline Altered mental status & $16(39.0)$ \\
\hline Hepatic dysfunction ${ }^{\mathrm{C}}$ & $4(9.8)$ \\
\hline Renal dysfunction $^{\mathrm{d}}$ & $12(29.3)$ \\
\hline Altered coagulation ${ }^{\mathrm{e}}$ & $6(14.6)$ \\
\hline Lactate $>2 \mathrm{mmol} / \mathrm{L}$ & $11(26.8)$ \\
\hline \multicolumn{2}{|l|}{ Source of infection } \\
\hline Genitourinary tract & $14(34.1)$ \\
\hline Respiratory tract & $2(4.9)$ \\
\hline Gastrointestinal tract & $9(22.0)$ \\
\hline Skin or soft tissue & $4(9.8)$ \\
\hline Endocarditis & $4(9.8)$ \\
\hline Primary BSI & $8(19.5)$ \\
\hline Septic shock & $6(14.6)$ \\
\hline Length of hospital stay (days) [median (IQR)] & $15(8-30)$ \\
\hline Alert from clinical microbiology laboratory & $20(48.8)$ \\
\hline In-hospital mortality & $10(24.4)$ \\
\hline
\end{tabular}

IQR, interquartile range; COPD, chronic obstructive pulmonary disease; SOFA, Sequential Organ Failure Assessment; $\mathrm{PaO}_{2}$, partial pressure of arterial oxygen; $\mathrm{FiO}_{2}$, percentage of inspired oxygen.

a Data are $n(\%)$ unless otherwise stated.

b Defined as systolic blood pressure (SBP) $<90 \mathrm{mmHg}$, mean arterial pressure $<70 \mathrm{mmHg}$ or a decrease in $\mathrm{SBP}>40 \mathrm{mmHg}$.

c Defined as bilirubin $>2 \mathrm{mg} / \mathrm{dL}$ or transaminases $>2 \times$ the upper limit of normal.

d Defined as urine output $<0.5 \mathrm{~mL} / \mathrm{kg} / \mathrm{h}$ for $\geq 2 \mathrm{~h}$ despite adequate fluid resuscitation or increase in creatinine $>0.5 \mathrm{mg} / \mathrm{dL}$ or $44.2 \mu \mathrm{mol} / \mathrm{L}$.

e Defined as international normalised ratio (INR) $>1.5$, activated partial thromboplastin time (aPTT) $>60 \mathrm{~s}$ or platelet count $<100 \times 10^{3} / \mu \mathrm{L}$.

identified. Previously known factors, such as presence of polymicrobial infection, age and SOFA score, were identified as risk factors for mortality. Moreover, a new peculiar variable, an early alert from the microbiology before the receipt of blood culture results, improved the outcome of these patients.

The most important finding of the current study is that effective communication between the clinical microbiology laboratory and prescribing physicians could promote early optimisation of antimicrobial regimens resulting in an improved outcome. In fact, we showed that receiving an early communication from the microbiology laboratory about the isolated pathogen allowed early modification of the treatment regimen. Since delay in delivery of appropriate antibiotic therapy is associated with higher mortality [18], receipt of an early communication from the laboratory could be essential in such cases. Processes involving communication between laboratories and clinical units are the most error-prone
Table 2

Comparison between survivors and non-survivors in patients with bloodstream infection (BSI) due to Enterococcus spp. ${ }^{\text {a }}$

\begin{tabular}{|c|c|c|c|}
\hline & $\begin{array}{l}\text { Survivors } \\
(N=31)\end{array}$ & $\begin{array}{l}\text { Non-survivors } \\
(N=10)\end{array}$ & $P$-value \\
\hline \multicolumn{4}{|l|}{ Demographic characteristics } \\
\hline Age (years) [median (IQR)] & $70(65-81)$ & $84.5(77.25-88)$ & $0.022^{*}$ \\
\hline Male sex & $14(45.2)$ & $6(60.0)$ & 0.414 \\
\hline Hospitalisation in last 3 months & $11(35.5)$ & $9(90.0)$ & $0.003^{*}$ \\
\hline $\begin{array}{l}\text { Antibiotic treatment in last } 3 \\
\text { months }\end{array}$ & $13(41.9)$ & $6(60.0)$ & 0.319 \\
\hline \multicolumn{4}{|l|}{ Means of acquisition } \\
\hline Community-acquired & $10(32.3)$ & 0 & $0.039^{*}$ \\
\hline Healthcare-associated & $10(32.3)$ & $7(70.0)$ & $0.035^{*}$ \\
\hline Nosocomial & $11(35.5)$ & $3(30.0)$ & 0.750 \\
\hline \multicolumn{4}{|l|}{ Co-morbid conditions } \\
\hline Cardiovascular disease & $20(64.5)$ & $8(80.0)$ & 0.360 \\
\hline Diabetes & $11(35.5)$ & $5(50.0)$ & 0.413 \\
\hline COPD & $7(22.6)$ & $4(40.0)$ & 0.280 \\
\hline Solid malignancy & $6(19.4)$ & $4(40.0)$ & 0.186 \\
\hline Chronic kidney disease & $7(22.6)$ & $2(20.0)$ & 0.864 \\
\hline Liver disease & $4(12.9)$ & $2(20.0)$ & 0.581 \\
\hline Neutropenia & - & $1(10.0)$ & 0.075 \\
\hline SOFA score [median (IQR)] & $2(1-4)$ & $11(6-14.25)$ & $<0.00^{*}$ \\
\hline $\begin{array}{l}\text { Charlson comorbidity index } \\
\text { [median (IQR)] }\end{array}$ & $3(2-4)$ & $4(3-8)$ & $0.049^{*}$ \\
\hline \multicolumn{4}{|l|}{ Source of infection } \\
\hline Genitourinary tract & $9(29.0)$ & $5(50.0)$ & 0.077 \\
\hline Respiratory tract & $2(6.5)$ & 0 & 0.410 \\
\hline Gastrointestinal tract & $8(25.8)$ & $1(10.0)$ & 0.294 \\
\hline Skin or soft tissue & $3(9.7)$ & $1(10.0)$ & 0.976 \\
\hline Endocarditis & $2(6.5)$ & $2(20.0)$ & 0.209 \\
\hline Primary BSI & $7(22.6)$ & $1(10.0)$ & 0.383 \\
\hline Septic shock & $3(9.7)$ & $3(30.0)$ & 0.114 \\
\hline $\begin{array}{l}\text { Alert from clinical microbiology } \\
\text { laboratory }\end{array}$ & $18(58.1)$ & $2(20.0)$ & $0.036^{*}$ \\
\hline \multicolumn{4}{|l|}{ Species distribution } \\
\hline Enterococcus faecalis & $21(67.7)$ & $7(70.0)$ & 0.894 \\
\hline Enterococcus faecium & $4(12.9)$ & 0 & 0.232 \\
\hline Other Enterococcus spp. & $3(9.7)$ & 0 & 0.307 \\
\hline Polymicrobial & $3(9.7)$ & $3(30.0)$ & 0.114 \\
\hline \multicolumn{4}{|l|}{ Antimicrobial susceptibility profile } \\
\hline Ampicillin-susceptible & $24(77.4)$ & $8(80.0)$ & 0.864 \\
\hline Ampicillin-resistant & $3(9.7)$ & $2(20.0)$ & 0.386 \\
\hline Vancomycin-resistant & $4(12.9)$ & 0 & 0.232 \\
\hline \multicolumn{4}{|c|}{ Definitive antimicrobial therapy } \\
\hline Ampicillin \pm AG & $9(29.0)$ & $2(20.0)$ & 0.575 \\
\hline $\mathrm{TZP} \pm \mathrm{AG}$ & $4(12.9)$ & 0 & 0.232 \\
\hline Carbapenem \pm AG & $9(29.0)$ & $3(30.0)$ & 0.953 \\
\hline Teicoplanin \pm AG & $2(6.5)$ & $3(30.0)$ & $0.048^{*}$ \\
\hline Vancomycin \pm AG & $3(9.7)$ & 0 & 0.307 \\
\hline Linezolid & $2(6.5)$ & 0 & 0.410 \\
\hline Others & $2(6.5)$ & $2(20.0)$ & 0.209 \\
\hline
\end{tabular}

$\mathrm{IQR}$, interquartile range; COPD, chronic obstructive pulmonary disease; SOFA, Sequential Organ Failure Assessment; AG, aminoglycoside; TZP, piperacillin/tazobactam.

a Data are $n(\%)$ unless otherwise stated.

Statistically significant $(P \leq 0.05)$

Table 3

Cox regression analysis of risk factors for in-hospital mortality in patients with bloodstream infection due to Enterococcus spp.

\begin{tabular}{lll}
\hline Risk factor & HR $(95 \% \mathrm{CI})$ & $P$-value \\
\hline Polymicrobial infection & $9.100(1.295-63.949)$ & $0.026^{*}$ \\
Age & $1.261(1.029-1.546)$ & $0.025^{*}$ \\
SOFA score & $1.244(1.051-1.474)$ & $0.011^{*}$ \\
Alert from clinical microbiology laboratory & $0.073(0.007-0.805)$ & $0.033^{*}$ \\
\hline
\end{tabular}

HR, hazard ratio; CI, confidence interval; SOFA, Sequential Organ Failure Assessment.

Statistically significant $(P \leq 0.05)$

parts of laboratory testing [28]. Blood culture results may remain pending for several days, but reporting significant test results to clinical staff by phone or in written form could be crucial to guide antimicrobial therapy. The timeliness and accuracy of preliminary blood culture reports could affect patient outcomes $[22,29]$. 
However, this procedure is often challenging. A recent study highlighted the key communication barriers between microbiology laboratories and clinical units [30]. Disruption at the interface between laboratories and clinical units, mutual lack of insight into each other's area of expertise, and limited laboratory services are all barriers that need to be addressed in order to improve communication [30]. For these reasons, the early alert from the microbiology laboratory to clinical units should represent one of the key points of antimicrobial stewardship programmes (ASPs). On one hand, establishing ASPs in hospitals can be an efficient framework to facilitate communication and to increase insight between the microbiology laboratory and clinical staff. On the other hand, including an intervention of early alert from the microbiology laboratory to clinical wards could guarantee the effectiveness of ASPs.

In the current study, in-hospital mortality of patients with BSI due to Enterococcus spp. was in line with previous reports in other hospital settings [2,17]. BSIs due to Enterococcus spp. are associated with different rates of mortality depending on the source of infection, with gastrointestinal sources showing the highest case fatality rates [2,31]. Similarly, infective endocarditis caused by Enterococcus spp. is responsible for high mortality [16]. Other factors, such as the presence of multiple co-morbidities, malignancy, diabetes, inflammatory bowel disease and cardiovascular failure, negatively affect the outcome of patients with enterococcal BSI $[12,16]$. Polymicrobial infection, age and SOFA score were identified as factors independently associated with mortality. It has recently been demonstrated that $E$. faecalis can facilitate co-infection with other micro-organisms, in particular with Escherichia coli, through the suppression of nuclear factor kappa-B (NF-кB)-driven responses in macrophages [32] and the increase of metabolic cue that facilitates E. coli growth [33]. Enterococcus spp. can also be found together with Candida spp. when there is translocation of intestinal pathogens from the gut into the bloodstream [31]. Besides, independently from the co-isolated pathogen, the presence of Enterococcus spp. as a part of a polymicrobial infection appears to increase the risk of short-term mortality [34].

This study has some limitations. First, the sample size is not so large, but slightly lower than other studies [12]. Second, only patients hospitalised in medical wards were included. Thus, these findings are not generalisable to other settings such as surgical wards or ICUs. Finally, the inclusion of polymicrobial infections could have influenced the study results. However, the six cases of polymicrobial infection were represented by BSI in which only one blood culture was positive for two micro-organisms whereas the subsequent cultures were positive only for Enterococcus spp., and all cases with the exception of one case of E. faecium-Candida coinfection received adequate antimicrobial therapy. The strengths of this study are that it is a multicentre study and that data were prospectively collected using standard procedures.

In conclusion, BSI due to Enterococcus spp. in elderly patients is associated with a high risk of in-hospital mortality. Presence of a polymicrobial infection, age and SOFA score on the day of BSI were factors associated with increased mortality. Conversely, an early alert from the microbiology laboratory before obtaining blood cultures results can help clinicians to select appropriate antimicrobial therapy, thus improving patient survival. Rapid and efficacious communication from the microbiology laboratory to clinical units is crucial and should be implemented in hospital settings.

\section{Funding}

None.

\section{Competing interests}

None declared.

\section{Ethical approval}

Not required.

\section{References}

[1] Low DE, Keller N, Barth A, Jones RN. Clinical prevalence, antimicrobial susceptibility, and geographic resistance patterns of enterococci: results from the SENTRY Antimicrobial Surveillance Program, 1997-1999. Clin Infect Dis 2001;32(Suppl. 2):S133-45.

2] Billington EO, Phang SH, Gregson DB, Pitout JD, Ross T, Church DL, et al Incidence, risk factors, and outcomes for Enterococcus spp. blood stream infections: a population-based study. Int J Infect Dis 2014;26:76-82.

[3] de Kraker ME, Jarlier V, Monen JC, Heuer OE, van de Sande N, Grundmann H. The changing epidemiology of bacteraemias in Europe: trends from the European Antimicrobial Resistance Surveillance System. Clin Microbiol Infect 2013;19:860-8.

[4] Eliopoulos GM. Increasing problems in the therapy of enterococcal infections. Eur J Clin Microbiol Infect Dis 1993;12:409-12.

[5] Willems RJ, Top J, van Santen M, Robinson DA, Coque TM, Baquero F, et al. Global spread of vancomycin-resistant Enterococcus faecium from distinct nosocomial genetic complex. Emerg Infect Dis 2005;11:821-8.

[6] Wisplinghoff H, Bischoff T, Tallant S, Seifert H, Wenzel RP, Edmond MB. Nosocomial bloodstream infections in US hospitals: analysis of 24,179 cases from a prospective nationwide surveillance study. Clin Infect Dis 2004:39:309-17.

[7] Caballero-Granado FJ, Becerril B, Cuberos L, Bernabeu M, Cisneros JM, Pachón J. Attributable mortality rate and duration of hospital stay associated with enterococcal bacteremia. Clin Infect Dis 2001:32:587-94.

[8] Orsi GB, Falcone M, Venditti M. Surveillance and management of multidrugresistant microorganisms. Expert Rev Anti Infect Ther 2011;9:653-79.

[9] Hidron AI, Edwards JR, Patel J, Horan TC, Sievert DM, Pollock DA, et al. NHSN annual update: antimicrobial-resistant pathogens associated with healthcareassociated infections: annual summary of data reported to the National Healthcare Safety Network at the Centers for Disease Control and Prevention, 2006-2007. Infect Control Hosp Epidemiol 2008;29:996-1011.

[10] Lee T, Pang S, Abraham S, Coombs GW. Antimicrobial-resistant CC17 Enterococcus faecium: the past, the present and the future. J Glob Antimicrob Resist 2018;16:36-47.

[11] McBride SJ, Upton A, Roberts SA. Clinical characteristics and outcomes of patients with vancomycin-susceptible Enterococcus faecalis and Enterococcus faecium bacteraemia-a five-year retrospective review. Eur J Clin Microbiol Infect Dis 2010;29:107-14.

[12] Bar K, Wisplinghoff H, Wenzel RP, Bearman GM, Edmond MB. Systemic inflammatory response syndrome in adult patients with nosocomial bloodstream infections due to enterococci. BMC Infect Dis 2006;6:145.

[13] Song X, Srinivasan A, Plaut D, Perl TM. Effect of nosocomial vancomycinresistant enterococcal bacteremia on mortality, length of stay, and costs. Infect Control Hosp Epidemiol 2003;24:251-6.

[14] Ong DS, Bonten MJ, Safdari K, Spitoni C, Frencken JF, Witteveen E, et al Epidemiology, management, and risk-adjusted mortality of ICU-acquired enterococcal bacteremia. Clin Infect Dis 2015;61:1413-20.

[15] Falcone M, Russo A, Venditti M. Optimizing antibiotic therapy of bacteremia and endocarditis due to staphylococci and enterococci: new insights and evidence from the literature. J Infect Chemother 2015;21:330-9.

[16] Pericàs JM, Cervera C, Moreno A, Garcia-de-la-Mària C, Almela M, Falces C, et al. Outcome of Enterococcus faecalis infective endocarditis according to the length of antibiotic therapy: preliminary data from a cohort of 78 patients. PLoS One 2018;13:e0192387 Erratum in: PLoS One 2018;13:e0196317.

[17] Poh CH, Oh HM, Tan AL. Epidemiology and clinical outcome of enterococcal bacteraemia in an acute care hospital. J Infect 2006:52:383-6.

[18] Fernández-Hidalgo N, Almirante B, Gavaldà J, Gurgui M, Peña C, de Alarcón A, et al. Ampicillin plus ceftriaxone is as effective as ampicillin plus gentamicin for treating Enterococcus faecalis infective endocarditis. Clin Infect Dis 2013;56:1261-8.

[19] Mazzone A, Dentali F, La Regina M, Foglia E, Gambacorta M, Garagiola E, et al. Clinical features, short-term mortality, and prognostic risk factors of septic patients admitted to internal medicine units: results of an Italian multicenter prospective study. Medicine (Baltimore) 2016;95:e2124.

[20] Falcone M, Tiseo G, Dentali F, La Regina M, Foglia E, Gambacorta M, et al. Predicting resistant etiology in hospitalized patients with blood cultures positive for Gram-negative bacilli. Eur J Intern Med 2018;53:21-8.

[21] US Centers for Disease Control and Prevention (CDC). CDC/NHSN surveillance definitions for specific types of infections. 2019. . [Accessed 5 July 2019] https://www.cdc.gov/nhsn/PDFs/pscManual/17pscNosInfDef_current.pdf.

[22] Bouza E, Sousa D, Muñoz P, Rodríguez-Créixems M, Fron C, Lechuz JG. Bloodstream infections: a trial of the impact of different methods of reporting positive blood culture results. Clin Infect Dis 2004;39:1161-9.

[23] Horan TC, Andrus M, Dudeck MA. CDC/NHSN surveillance definition of health care-associated infection and criteria for specific types of infections in the acute care setting. Am J Infect Control 2008;36:309-32.

[24] Friedman ND, Kaye KS, Stout JE, McGarry SA, Trivette SL, Briggs JP, et al. Health care-associated bloodstream infections in adults: a reason to change the accepted definition of community-acquired infections. Ann Intern Med 2002; 137:791-7. 
[25] Freifeld AG, Bow EJ, Sepkowitz KA, Boeckh MJ, Ito JI, Mullen CA, et al. Clinical practice guideline for the use of antimicrobial agents in neutropenic patients with cancer: 2010 update by the Infectious Diseases Society of America. Clin Infect Dis 2011:52:e56-93.

[26] National Kidney Foundation. K/DOQI clinical practice guidelines for chronic kidney disease: evaluation, classification, and stratification. Am J Kidney Dis 2002;39(2 Suppl. 1):S1-266.

[27] Magiorakos AP, Srinivasan A, Carey RB, Carmeli Y, Falagas ME, Giske CG, et al. Multidrug-resistant, extensively drug-resistant and pandrug-resistant bacteria: an international expert proposal for interim standard definitions for acquired resistance. Clin Microbiol Infect 2012;18:268-81.

[28] Hawkins R. Managing the pre- and post-analytical phases of the total testing process. Ann Lab Med 2012;32:5-16.

[29] Schifman RB, Meier FA, Souers RJ. Timeliness and accuracy of reporting preliminary blood culture results: a College of American Pathologists Q-probes study of 65 institutions. Arch Pathol Lab Med 2015;139:621-6.
[30] Skodvin B, Aase K, Brekken AL, Charani E, Lindemann PC, Smith I. Addressing the key communication barriers between microbiology laboratories and clinical units: a qualitative study. J Antimicrob Chemother 2017;72:2666-72.

[31] Falcone M, Russo A, Iraci F Carfagna P Goldoni P, Vullo V et al. Risk factors and outcomes for bloodstream infections secondary to Clostridium difficile infection. Antimicrob Agents Chemother 2015;60:252-7.

[32] Tien BYQ, Goh HMS, Chong KKL, Bhaduri-Tagore S, Holec S, Dress R, et al. Enterococcus faecalis promotes innate immune suppression and polymicrobial catheter-associated urinary tract infection. Infect Immun 2017;85: pii: e00378-17.

[33] Keogh D, Tay WH, Ho YY, Dale JL, Chen S, Umashankar S, et al. Enterococcal metabolite cues facilitate interspecies niche modulation and polymicrobial infection. Cell Host Microbe 2016;20:493-503.

[34] Ceci M, Delpech G, Sparo M, Mezzina V, Sánchez Bruni S, Baldaccini B. Clinical and microbiological features of bacteremia caused by Enterococcus faecalis. J Infect Dev Ctries 2015;9:1195-203. 